\title{
Partial repetition costs persist in nonsearch compound tasks: Evidence for multiple-weighting-systems hypothesis
}

\author{
Michael Zehetleitner • Dragan Rangelov • \\ Hermann J. Müller
}

Published online: 3 March 2012

(C) Psychonomic Society, Inc. 2012

\begin{abstract}
Search performance is sequence-dependent. A specific finding observed in compound-search tasks consists of an interaction between cross-trial sequences (repetition vs. change) of the target-defining (primary) and responsedefining (secondary) features: The effect of a target change is greater when the response stays the same than when the response changes. The present study tested whether this interaction arises from processes involved in target search or from later processes in compound tasks. Uncertainty about the upcoming target location - that is, the search component of compound tasks - was removed in different experiments, either by the use of exogenous spatial precues or by presenting only one, central item. Despite having removed the search component, we observed a robust interaction between target (primary) and response (secondary) feature sequences. These results suggest that this interaction originates from a processing stage concerned with discriminating the response feature of a single (selected) item, rather than from a search-related stage. Furthermore, the results support our multiple-weighting-systems hypothesis, according to which sequence effects in visual search tasks do not stem from a single, unitary mechanism; rather, multiple stages of processing on any given trial can lead to separate memory traces, which in turn have effects on different stages of processing on the subsequent trial.
\end{abstract}

M. Zehetleitner $(\bowtie) \cdot$ D. Rangelov $\cdot$ H. J. Müller

Ludwig-Maximilians-Universität München,

General and Experimental Psychology,

Leopoldstr. 13,

80802 München, Germany

e-mail: mzehetleitner@psy.lmu.de

H. J. Müller

Birkbeck College, University of London,

London, UK
Keywords Attention · Interactions with memory - Visual search $\cdot$ Repetition effects

In visual search, performance on a given trial is frequently modulated both by the dimensions and features of the stimuli encountered on the preceding trial and by the actions performed in response to them. That is, search performance is modulated by the match between consecutive trials in terms of critical stimulus and response attributes (e.g., Found \& Müller, 1996; Kristjánsson \& Campana, 2010; Lamy, Yashar, \& Ruderman, 2010; Maljkovic \& Nakayama, 1994). Such intertrial effects are usually explained in terms of implicit-memory traces established by processing on a given trial $n$, which then influence (or "prime") the processing on the subsequent trial $n+1$. However, what is controversial is whether the effects of implicit memory in visual search affect early, preattentive stages of processing (e.g., Found \& Müller, 1996; Goolsby \& Suzuki, 2001; Zehetleitner \& Müller, 2010) or late, postselective stages (e.g., Mortier, Theeuwes, \& Starreveld, 2005). The standard view is to assume that one unitary memory system is responsible for intertrial priming effects in visual search (e.g., Becker, 2010; Hillstrom, 2000; Huang, Holcombe, \& Pashler, 2004).

\section{Multiple weighting systems}

In contrast to single-mechanism explanations, a multipleweighting-system (MWS) account has recently been proposed that assumes that processing at multiple stages (e.g., target selection, response selection) on any given trial $n$ can lead to the laying down of separate, stage-specific memory traces that in turn influence the processing on the respective 
stages on the subsequent trial $n+1$ (Ásgeirsson \& Kristjánsson, 2011; Kristjánsson \& Campana, 2010; Lamy et al., 2010; Rangelov, Müller, \& Zehetleitner, 2011a, 2011b, in press). Specifically, Töllner, Gramann, Müller, Kiss, and Eimer (2008) noted that, prior to their study, the problem had been ill-posed, because asking whether the effect of implicit memory in visual search is preattentive or postselective in origin assumes that only one such effect operates, either early or late (an assumption that has been explicitly made by, e.g., Hillstrom, 2000; Huang et al., 2004; and Becker, 2010). Instead, as Töllner et al. argued, the observable behavioral intertrial effects are, in fact, a superposition of several cognitive-sequence effects that operate at different levels, or stages, in the processing hierarchy, and whose underlying mechanisms are independent of each other (see Rangelov et al., in press, for more details).

The transition from models assuming a single, unitary weighting mechanism to the MWS account offers a novel, fruitful theoretical framework to investigate intertrial effects in visual search. More than in earlier accounts, a thorough conceptual analysis is necessary, including a differentiation between task requirements and the cognitive processes necessary to solve the task. In the present study, we present one such task microanalysis.

The number of stages involved in cognitive processing depends on the task requirements (Rangelov et al., 2011a, b, in press). For instance, a compound-search task consists of search, discrimination, and response components (see also Huang et al., 2004; Töllner et al., 2008; and the present Fig. 1). In a compound task, a target is presented among nontargets and is defined by, for instance, a unique color or form (the primary feature; see Fig. 2). Observers' task is to discriminate and report a secondary visual feature of the target stimulus (e.g., the orientation, horizontal vs. vertical, of the target's stripes; see Fig. 2).

In general, there are two different levels of description for compound tasks: the levels of task requirements and cognitive processes (see Figs. 1a and 1b, respectively). Frequently, this conceptual distinction is not relevant, as the two types of description can be used interchangeably. For the present study, however, the distinction between task components and the requisite cognitive processes is of central importance. With respect to task requirements, the label "compound" implies that the task consists of two components: a search and a discrimination component. The target has first to be searched for, on the basis of its primary visual feature, and then its secondary, response-defining feature has to be discriminated. In terms of cognitive processes, the search component requires, first, the singling out of an item from amongst other items on the basis of salience (attentional selection) and, second, the identification of this item as a target or nontarget (see Wolfe, 1994) on the basis of the target-defining primary visual features (Bravo \& Nakayama, 1992; Duncan, 1985). The discrimination component involves identification of the response-defining, secondary visual feature and mapping this feature onto the correct response (response selection). Finally, the response is planned and executed. Thus, importantly, two identification processes take place: one directed to the primary visual feature, to verify the selected item's status as a target (search component), and another directed to the secondary visual feature, to determine the appropriate response (discrimination component).

\section{Partial-repetition costs}

These theoretical considerations are necessary to describe and explain a specific type of intertrial effect that is frequently observed in compound-search tasks: partial-repetition costs (PRCs). PRCs consist of a specific pattern of interaction between repetitions or changes of the target- and responsedefining features (i.e., the primary and secondary features): Reaction times (RTs) are fast when the target- and responsedefining features both repeat or both change, but are slow when one feature repeats and the other changes (Chan \& Hayward, 2009; Hillstrom, 2000; Huang et al., 2004; Lamy et al., 2010; Müller \& Krummenacher, 2006; Olivers \& Meeter, 2006). Two competing explanations have been

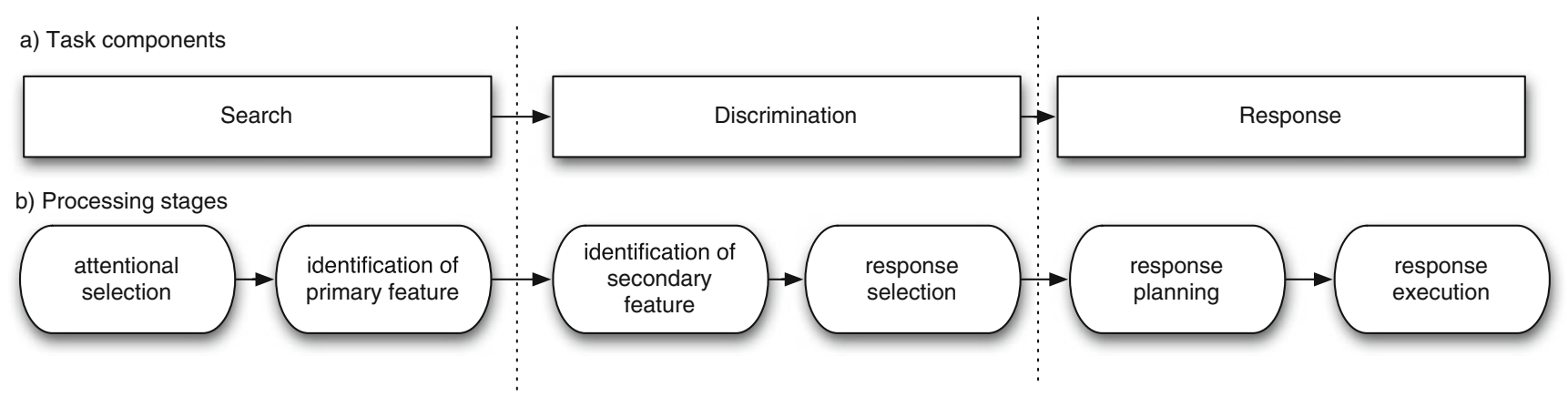

Fig. 1 Panels a and b, respectively, represent the task components and the corresponding processing stages required for solving a standard compoundsearch task, assuming a serial processing architecture (see, e.g., Wolfe,
1994). Note that parallel architectures (e.g., Bundesen, 1990)—which, however, are infrequently mentioned in the literature discussion of intertrial effects - would have to be depicted differently 
Fig. 2 Example displays for Experiments 1a (left panel) and $1 \mathrm{~b}$ (right panel)
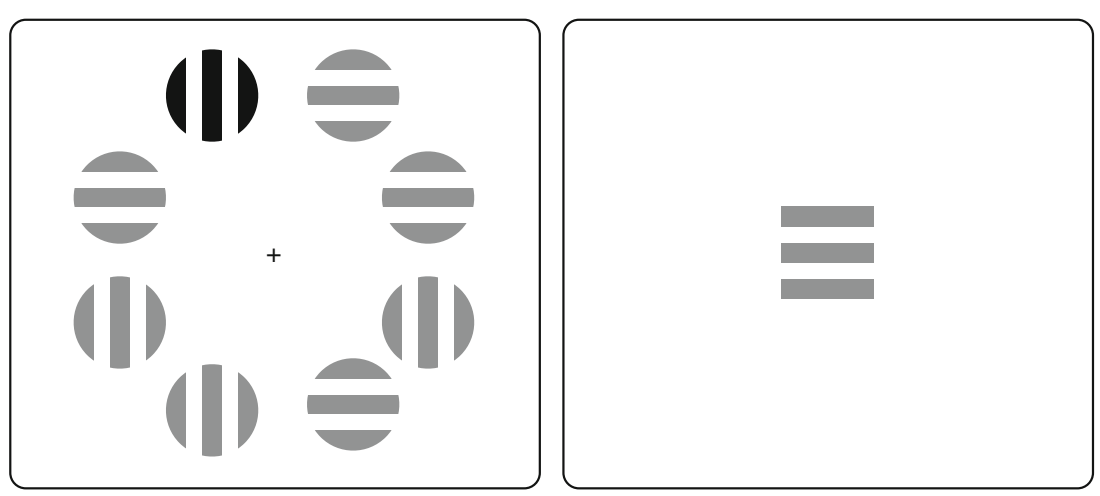

proposed to account for such PRCs: Huang et al. assumed that PRCs arise from the search component-specifically, from the target-versus-nontarget verification process-in compound tasks; by contrast, Töllner et al. (2008) assumed that PRCs arise from the subsequent discrimination componentspecifically, from determination of the reaction-critical feature and mapping it onto the appropriate response. Huang et al. found an overall RT advantage for repeating versus changing the target-defining feature. In addition, changes of the targetand response-defining features interacted: Target repetitions were faster than target changes, but only if the responsedefining (secondary) feature repeated as well. To explain this pattern, Huang et al. proposed an "episodic-retrieval account," according to which the (search) process of verifying that the selected item is indeed the target is modulated by its match to an episodic-memory trace of the previously selected target: That is, verification is faster if the currently selected item and the previous target are the same or different in their primary as well as secondary visual features, but verification is slower if one feature is the same but the other is different. Note that establishing a match for the target-defining feature is assumed to explain the main effect of repeating the primary feature. However, the duration (but not the outcome) of the verification process is also modulated by a match/mismatch in the response-defining feature, because "the matching process is not successfully confined to the [primary] dimension" (Huang et al., 2004, p. 20). PRCs are then the result of "inconsistency across dimensions": "When it arises, the system may need extra time to double check the status of the target" (p. 20; see also the present Fig. 1).

Töllner et al. (2008) also found PRCs, in addition to main effects of repeating versus changing the target- and responsedefining features. However, in contrast to Huang et al. (2004), they proposed that the PRCs stem from the discrimination component of compound tasks - specifically, from the determination of the secondary, response-defining feature (or the mapping of this feature onto its associated response). This conclusion was based on electrophysiological evidencenamely, that an electrophysiological marker of attentional selection, the N2pc (see, e.g., Eimer, 1996), was affected exclusively by changes (vs. repetitions) of the target-defining feature, whereas a marker of response production, the response-locked lateralized readiness potential or rLRP (Hackley \& ValleInclán, 2003), was affected only by changes (vs. repetitions) of the response-relevant feature. In order to explain the interaction between changes (vs. repetitions) of the responserelevant and target-defining features, Töllner et al. assumed that the discrimination component of compound tasks is fast if both the target and the response features repeat or both change, but slow if one feature changes and the other repeats, in line with the idea of "linked expectancies" (cf. Kingstone, 1992): If the target-defining feature is found to repeat/change, the system implicitly "expects" the response-defining feature to repeat/change too; in all other cases, extra time is taken to discriminate the response-defining feature (and/or to select the associated response).

\section{Purpose of the present study}

In order to apply the analysis suggested by the MWS account to the phenomenon of PRCs and to differentiate between the competing theoretical explanations, we here present three experiments (1a, 1b, and Experiment 2), as well as an arithmetical simulation of interactions between the different processing stages leading to the PRC. The present Experiment Experiment 1 was designed to establish whether PRCs originate from the search or the discrimination component of compound tasks, thereby permitting a decision between the two competing accounts. To this end, we introduced two methods to eliminate the search component from the compound task in Experiments $1 \mathrm{a}$ and $1 \mathrm{~b}$. In Experiment 1a, we adapted the cueing-search paradigm of Goolsby and Suzuki (2001) to the dimension change paradigm. That is, observers performed a standard compound task. From trial to trial, the target-defining dimension (primary feature) could either change or repeat. Additionally, on half of all trials, an exogenous spatial cue indicated the target position prior to the onset of the search display. This way, on search trials there was uncertainty about the target position, which observers had to resolve by searching for it; by contrast, on nonsearch trials there was no uncertainty about the target position, and thus search was not necessary. 
However, according to Huang et al. (2004), because observers had to verify the target identity on search trials, they might also have verified the identity of the cued item on nonsearch trials (is it indeed the target?), even though the cue was 100\% valid, so as to avoid reconfiguring the task set across standard and cued trials. For this reason, in Experiment 1b, we removed the search component of compound tasks completely by presenting just one, single item at a constant location.

With regard to the interaction between changes (vs. repetitions) of the primary and secondary features in the compound task, the two competing accounts of PRCs make differential predictions: When the search component of compound tasks is removed, the selection account of Huang et al. (2004) predicts that no such interaction will occur; this is in contrast to the response selection account of Töllner et al. (2008), according to which one would still expect the interaction to be manifest. To anticipate the results, they were in line with the response selection account.

Experiment 2 was designed to investigate more precisely which response-related processing stage PRCs stem from: specifically, whether PRCs depend on the sequence of the secondary feature (discrimination) or of the response (motor response). To examine this question, we adapted a paradigm developed by Yashar and Lamy (2011), who addressed the same question for the feature-priming paradigm (Maljkovic \& Nakayama, 1994): Instead of presenting just two secondary, response-relevant features (e.g., vertical and horizontal orientation), they presented four secondary features, two of which were mapped to one response, the other two of which were mapped to the alternative. In this way, it became possible to disentangle the effects of response repetition and response feature repetition. The results favored the former being the critical factor.

Note that in the literature, as well as in the present study, the interaction between the sequence of the primary visual feature and the sequence of the secondary feature or of the (selected/executed) response can take on different forms in an interaction plot (see Fig. 5 below for an illustration of typical patterns). To understand how these different kinds of interactions can actually be generated without assuming different shapes of the (theoretical) PRCs, we go on to present a schematic mathematical description of the MWS account that permits inferences to be drawn as to the sources of the interaction patterns.

\section{Experiment 1}

In Experiment 1, two methods were employed to remove the search component from compound tasks in either a portion of trials (Exp. 1a) or all trials (Exp. 1b). For Experiment 1a, we used a standard compound task, in which a circular array of eight stimuli, consisting of seven nontargets (blue circles) and one target (blue square or green circle, serving as a form or a color target, respectively), were presented on each trial. Each stimulus contained a vertical or horizontal grating, and observers had to respond to the orientation of the target grating. That is, the primary (target-defining) feature was the stimulus color or form, and the secondary (responsedefining) feature was the stimulus orientation. In Experiment $1 \mathrm{a}$, on $50 \%$ of all trials, the target location was exogenously cued (with $100 \%$ validity) before display onset, removing the need to search for the target on such "nonsearch" trials. In Experiment 1b, nontargets (blue circles) were removed from the displays altogether, and the target was always presented at the center of the screen. Observers' task was again to indicate the orientation of the target's stripes. In contrast to Experiment 1a, in which color and form were task-relevant on half of the trials, because they indicated the target's location, in Experiment $1 \mathrm{~b}$ they were completely irrelevant for the task. That is, the single items presented in Experiment $1 \mathrm{~b}$ were physically equivalent to the targets in the compound-search task of Experiment 1a as well as in standard compound tasks (as used in, e.g., Töllner et al., 2008), but the primary visual feature - on the basis of which, in the standard search task, a selected item would be identified as the response-relevant target (rather than a nontarget) - was completely irrelevant.

In both experiments, we examined whether changes (vs. repetitions) of the target's response-defining feature (i.e., the stripes' orientation) would interact with featural changes of the target's primary feature (i.e., the target's color/shape). The search and discrimination accounts of PRCs make differential predictions regarding the interaction between changes (vs. repetitions) of the color/form and the (stripe) orientation of the target in the present task. Huang et al. (2004) would predict that no such interaction should occur because, in the present task, the search component was eliminated. Consequently, there was no longer a need to verify that the single item presented was a target (it was invariably a target!) - which is the process Huang et al. assumed PRCs to derive from in the compound-search task. By contrast, the finding of PCRs in nonsearch trials of Experiment 1a as well as in the trials of Experiment $1 \mathrm{~b}$ would be consistent with Töllner et al. (2008) because, although no search was required, there was still a need to determine the response-defining feature (and to map it onto the associated response) - that is, the discrimination component — which, on their account, is the origin of PCRs.

\section{Method}

Participants A group of 11 observers took part in Experiment 1a (4 female, 7 male; mean age 26 years). Another 11 observers participated in Experiment $1 \mathrm{~b}$ ( 7 female, 4 male; mean age 
25 years). All had normal or corrected-to-normal vision, were naïve with respect to the purpose of the experiment, and were paid $€ 8 / \mathrm{h}$ for participating.

Apparatus The experiments were controlled by a PC running under Windows XP. The stimuli were presented on a 21 -in. CRT monitor $(1,280 \times 1,024$ pixels resolution; 100 $\mathrm{Hz}$ refresh rate). The viewing distance was $57 \mathrm{~cm}$, controlled by a chinrest. The participants responded by pressing the left or the right button of a computer mouse with their left or right index finger, respectively.

Stimuli and procedure The stimuli were blue (CIE .257, .310, 30 ) or green (CIE $.315, .518,27)$ circles or squares that could either be horizontally or vertically striped (see Fig. 2), subtending $2^{\circ} \times 2^{\circ}$ of visual angle (the circles had a diameter of $2^{\circ}$ ). The targets in Experiments 1a and $1 \mathrm{~b}$ were equally likely to be a blue square or a green circle. The nontargets in Experiment 1a were all blue circles. In this experiment, the display consisted of a circular arrangement of eight stimuli (seven nontargets and one target) with a radius of $4^{\circ}$ from the center of the screen. In Experiment $1 \mathrm{~b}$, a single target was presented at the center of the screen (see Fig. 2).

In Experiment 1a, a perfectly valid cue indicated the target location on $50 \%$ of all trials. As in Goolsby and Suzuki (2001), the cue consisted of an outline box with white line color. This box was presented for $20 \mathrm{~ms}$ and followed by a blank screen for $120 \mathrm{~ms}$, after which the search display appeared. On noncued trials, the fixation cross was presented for an additional $140 \mathrm{~ms}$ to equate the intervals between the start of the trial and onset of the search display for cued and noncued trials.

Observers' task was to discriminate the orientation of the object's grating (stripes) by a keypress. Different stimulusto-response mappings were counterbalanced across participants. The stimuli were presented until a response was made, and trials were separated by a variable interval between the response and the onset of the next trial. Following a correct response, only a fixation point was visible on the screen during this interval (700 to $1,200 \mathrm{~ms}$ ). Erroneous responses were followed by an empty (black) screen $(1,000 \mathrm{~ms})$.

Design The orientation of the inner grating (i.e., the secondary, response-relevant feature) could either repeat or change across consecutive trials. Independently of this, the color or form (i.e., the primary, target-defining feature) could either repeat or change across trials. Note that we will refer to the target's color and form as the "primary feature" throughout the remainder of the article, even though color and form were target-defining only in Experiment 1a. In Experiments $1 \mathrm{~b}$ and Experiment 2, the target's color and form were irrelevant for performing the task - namely, to respond to the orientation of the grating of the single item presented in the display. That is, the primary feature was target-defining in the standard search trials of Experiment 1a and an irrelevant attribute in the nonsearch trials of Experiment 1a and in all trials of Experiments $1 \mathrm{~b}$ and Experiment 2.

This resulted in a basic $2 \times 2$ experimental design with the factors Sequence of the Primary Feature and Sequence of the Secondary Feature (two levels for both: repetition vs. change) across consecutive trials. In Experiment 1a, an additional factor was Presence of the Target Location Cue.

\section{Results}

Participants made errors on approximately $5.6 \%$ and $4.0 \%$ of the trials in Experiments 1a and 1b, respectively. Two of the participants, one in Experiment 1a (10\%) and one in Experiment $1 \mathrm{~b}(8 \%)$, made considerably more errors than the others, which led to them being excluded from the subsequent analyses. Since inspection of the error pattern showed no indications of a speed-accuracy trade-off, errors were not analyzed further. Correct-response trials were then filtered for extreme RTs (faster than $150 \mathrm{~ms}$ and slower than $1,000 \mathrm{~ms}$, resulting in the loss of about $1 \%$ of the data) prior to analysis.

Experiment $1 a$ Individual participants' mean RTs were examined in a repeated measures analysis of variance (ANOVA) with the main terms primary-feature sequence (repetition vs. change) across trials, secondary- (responsedefining) feature sequence (repetition vs. change) across trials, and cue (present vs. absent).

The ANOVA revealed a significant a main effect of cue, $F$ $(1,10)=20.64, p<.01, \eta_{\mathrm{p}}{ }^{2}=.81$ : RTs were markedly faster on cued than on noncued trials (589 vs. $632 \mathrm{~ms}$ ), indicating that the cueing manipulation was successful (see Fig. 3). Furthermore, the main effect of primary-feature sequence was significant: RTs were faster for repetitions than for changes of the target-defining feature (656 vs. $691 \mathrm{~ms}), F(1,10)=12.1, p<.01, \eta_{\mathrm{p}}{ }^{2}=.55$. Importantly, there was an interaction between primary- and secondaryfeature sequence, $F(1,10)=12.3, p<.01, \eta_{\mathrm{p}}{ }^{2}=.55$, which did not interact with cue, $F(1,10)=0.8, p=.38, \eta_{\mathrm{p}}{ }^{2}=.07$. That is, there was a pattern of PRCs, and this pattern was unaffected by whether the task did (noncued trials) or did not (cued trials) require a search component. The presence of PRCs in the latter condition was confirmed by an ANOVA carried out on cue trials only, which also yielded a significant interaction of primary- and secondary-feature sequence, $F(1,10)=6.3, p<.05, \eta_{p}{ }^{2}=.38$. Additionally, in the overall ANOVA, we found a significant interaction 
a)

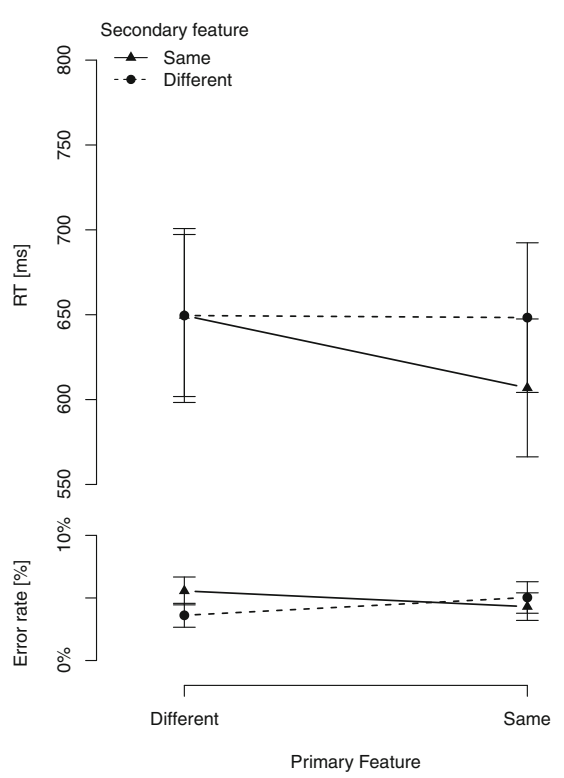

b)

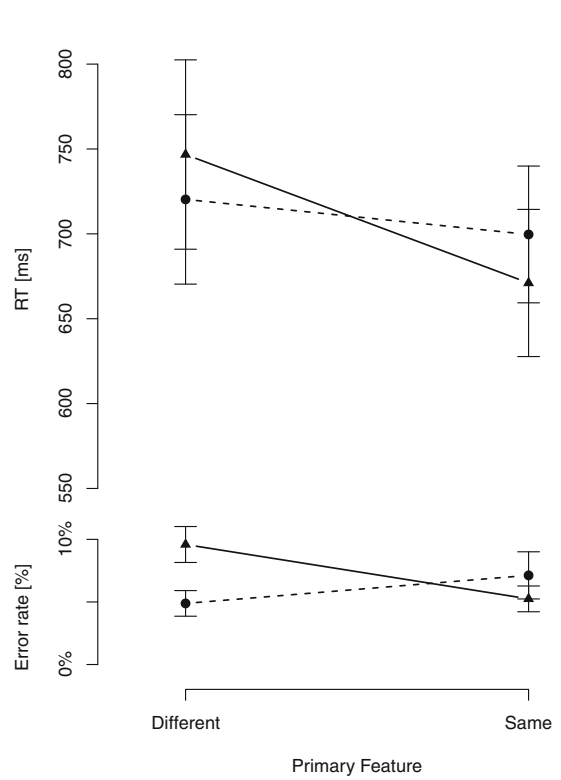

c)

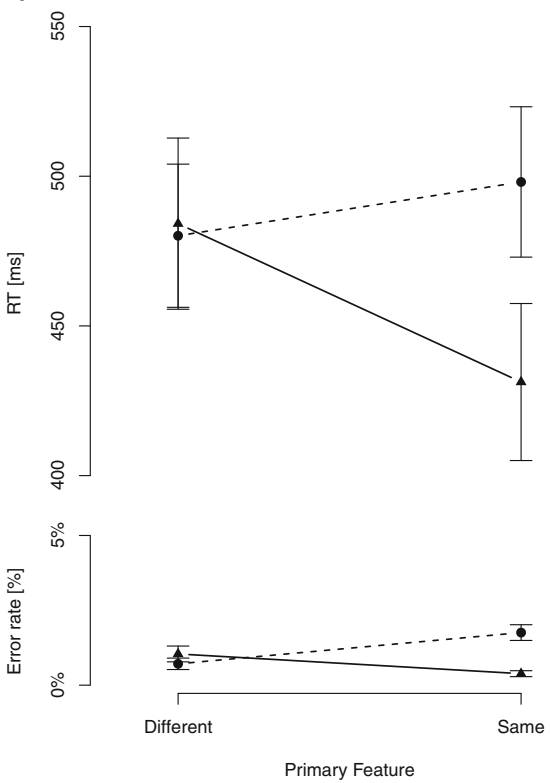

Fig. 3 Mean correct RTs and percentages of errors of Experiment Experiment 1, as a function of changes in the primary and secondary features of the stimuli. (Left) Cued trials in Experiment 1a. (Middle) Uncued trials in Experiment 1A. (Right) Experiment 1b. Vertical lines denote standard errors

between primary-feature sequence and cue: Dimension repetition (vs. change) effects were larger on search than on nonsearch trials (48 vs. $21 \mathrm{~ms}$ ). Post-hoc $t$ tests revealed the effects of both primary- and secondary-feature change to be significantly greater than zero (both $t \mathrm{~s}>2.4$, both $p \mathrm{~s}<.05$ ).

Experiment $1 b$ The ANOVA with the main terms primaryfeature sequence (repetition vs. change) and secondary-feature sequence (repetition vs. change) revealed both main effects to be significant: primary-feature sequence, $F(1,10)=30.79$, $p<.01, \eta_{\mathrm{p}}^{2}=.75 ;$ secondary-feature sequence $F(1,10)=$ $21.61, p<.01, \eta_{\mathrm{p}}{ }^{2}=.68$. Participants responded faster when the stimulus repeated than when it changed across trials (465 vs. $482 \mathrm{~ms}$ ). Additionally, they were faster to respond when the required response was the same as on the previous trial, rather than when it changed (458 vs. $489 \mathrm{~ms}$ ). The interaction between the primary- and secondary-feature sequences (illustrated in Fig. 3) also turned out to be significant, $F(1,10)=57.68, p<.01, \eta_{\mathrm{p}}{ }^{2}=.85$; that is, there was a pattern of PRCs.

\section{Discussion}

In summary, significant PRCs were obtained in both Experiments $1 \mathrm{a}$ and $1 \mathrm{~b}$, independently of whether or not the (compound) tasks involved a search component (i.e., of whether or not observers were required to search for the target). This is inconsistent with the account of Huang et al.
(2004), according to which PRCs would be expected to manifest only on the search trials of Experiment 1a. By contrast, it is consistent with the account of Töllner et al. (2008), which predicted that PRCs would occur in both search and nonsearch conditions.

\section{Experiment 2}

Experiments $1 \mathrm{a}$ and $1 \mathrm{~b}$ showed a significant interaction between primary- and secondary-feature sequences in "compound" tasks, producing PRCs whether or not observers had to search for the target. Additionally, Experiment $1 \mathrm{~b}$ demonstrated that PRCs were obtained even if the primary feature was not relevant for performing the task. Thus, taken together, our results are consistent with the idea that PRCs do not stem from the search but from the discrimination component of compound tasks, and can even be observed in nonsearch tasks. However, in Experiment Experiment 1, changes of the secondary, response-defining feature (vertical/horizontal stripes) were always associated with changes of the response (left/right buttonpress), as is the case in almost all compound-task experiments in the literature. Consequently, PRCs might stem from either the secondary-feature identification or response selection stages of the discrimination component (see Fig. 1). Recently, Yashar and Lamy (2011), using the feature-priming paradigm, demonstrated that the change of the motor response, and not only the change of the response-defining feature, is what generates PRCs. In their study, observers performed a compound task in which the primary, target-defining feature 
could change from trial to trial (more precisely, the colors of the target and the nontargets could be swapped from trial to trial). Importantly, the secondary, response-defining feature consisted of four (instead of only two) feature values, two of which were mapped to one response and the other two of which were mapped to the alternative response. Thus, on trials on which the response was the same as on the previous trial (response repetition), the secondary feature was equally likely to be either the same (repetition) or different (change), relative to the response-defining feature on the previous trial. However, such changes of the secondary feature alone (while the response repeated) did not significantly interact with changes of the primary, target-defining feature, indicating that PRCs stem from repetition of the motor response rather from repetition of the secondary feature. Experiment Experiment 2 was designed to investigate whether this finding generalizes to PRCs in the present nonsearch task. To this end, in Experiment Experiment 2, we examined the role of changes of the response, as contrasted with changes of the response-defining feature. The task was the same as in Experiment 1b, with the search component of compound tasks removed by presenting only one item in the center of the screen. Similarly to Yashar and Lamy, we introduced four response-relevant features that could change from trial to trial; two of these were mapped to one response, and the two, to the other response.

\section{Method}

Participants A group of 15 observers took part in Experiment Experiment 2 ( 10 female, 5 male; mean age 26 years). All had normal or corrected-to-normal vision, were naïve with respect to the purpose of the experiment, and were paid $€ 8 / \mathrm{h}$ for participating.

Apparatus The apparatus was identical to that of Experiment Experiment 1.

Stimuli and procedure The stimuli and procedure were identical to those of Experiment 1b, except that the target could be striped in orientations tilted $\pm 10^{\circ}$ and $\pm 80^{\circ}$ from the vertical. Stimuli tilted to the left required a left-hand response, and stimuli tilted to the right required a right-hand response.

Design The experiment consisted of seven blocks of 80 trials each. On each trial, the primary visual feature (color/ form) could repeat or change relative to the previous trial. Additionally, the response could repeat or change. If the response changed, the secondary, response-relevant feature also changed. If the response repeated, the secondary feature could either repeat or change.

\section{Results}

Error rates were low (3.1\%), and there was no indication of a speed-accuracy trade-off. Prior to the RT analysis, responses faster than $150 \mathrm{~ms}$ and slower than $1,500 \mathrm{~ms}$ were eliminated (1.4\% of all trials). Analogously to Yashar and Lamy (2011), we first analyzed the interactions between several types of repetition: of the primary feature and the motor response; second, of the primary feature and the secondary, response-defining feature; and finally, of the primary feature and the combination of the secondary feature and the response (the "usual" interaction studied; see Fig. 4). As proposed by Yashar and Lamy, these three types of interaction permit conclusions as to the processing stage at which PRCs arise: the motor stage, given that significant PRCs are revealed by the first analysis (in which only the sequence of motor responses is considered), or the discrimination stage, given that significant PRCs are revealed by the second analysis (in which the response is constant and only the sequence of the secondary feature is considered).

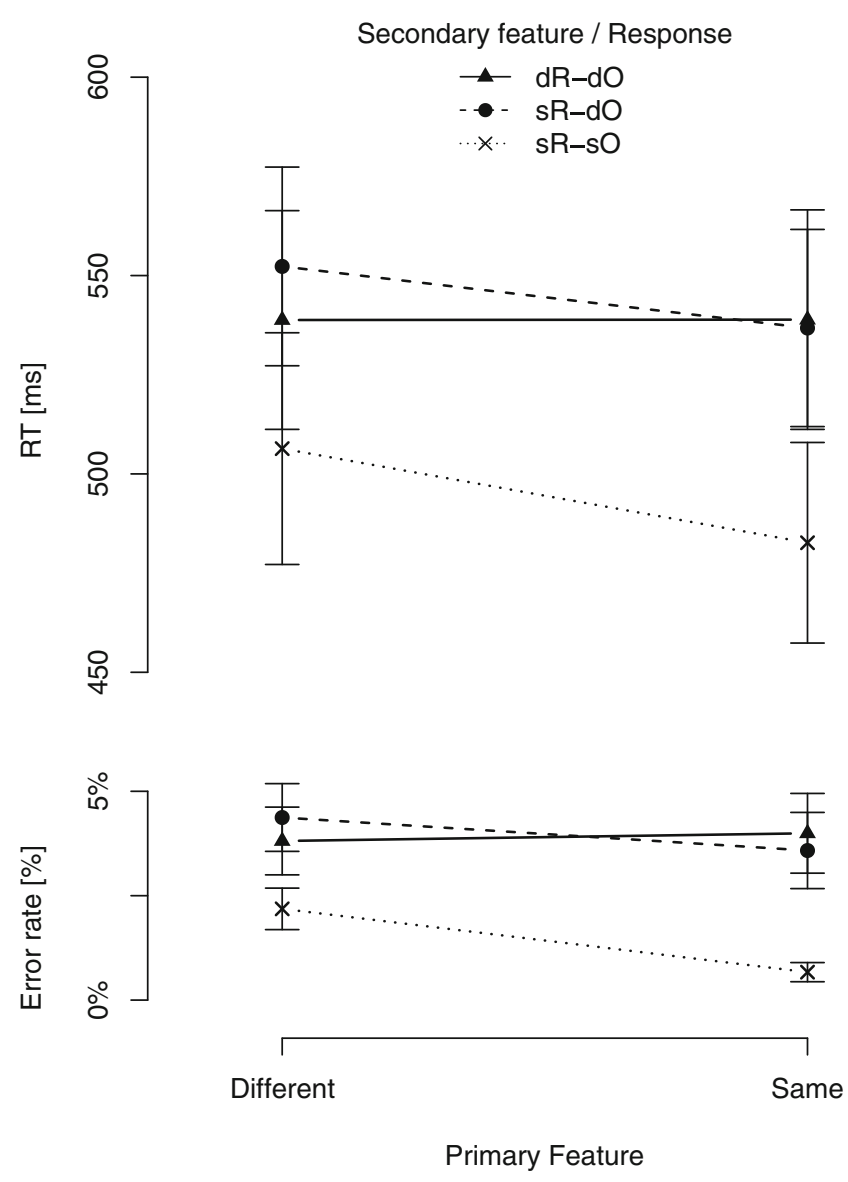

Fig. 4 Mean correct RTs and percentages of errors for Experiment Experiment 2, as a function of changes in the primary feature and the secondary feature/response. In the legend, "d" and "s" stand for "different" and "same," and "R" and "O" stand for "response" and "orientation." Vertical lines denote standard errors 
The third analysis corresponds to the interaction usually computed in studies with only two secondary features, so that changes of the secondary feature always coincide with changes of the response.

Motor response repetition For this analysis, we selected only trials on which the orientation of the stimulus (the secondary feature) changed relative to the previous trial, and then examined the effect of response sequence (repetition vs. change) dependent on the primary-feature sequence (repetition vs. change). An ANOVA with these two factors revealed a significant main effect of primary-feature sequence, $F(1,14)=10.1$, $p<.01, \eta_{\mathrm{p}}{ }^{2}=.42$ : RTs were faster on trials on which the color/ form of the stimulus repeated, as compared to trials on which the color/form changed (537 vs. $545 \mathrm{~ms}$ ). The main effect of response sequence was nonsignificant, $F(1,14)=0.18, p=.68$. Of theoretical importance, the interaction was significant, $F(1$, 14) $=4.9, p<.05, \eta_{\mathrm{p}}{ }^{2}=.26$, indicating that repetition of the motor response was crucial for PRCs to arise.

Response feature repetition For response repetition trials, an ANOVA with the factors Change Versus Repetition of the Primary Feature (color/form) and Change Versus Repetition of the Secondary, Response-Defining Feature (orientation) revealed both main effects to be significant: $F(1,14)=21.6$, $p<.001, \eta_{\mathrm{p}}{ }^{2}=.61$, and $F(1,14)=25.4, p<.001, \eta_{\mathrm{p}}{ }^{2}=.64$, respectively. Changes of the primary feature led to slower RTs relative to repetitions ( 509 vs. $530 \mathrm{~ms}$ ), and changes of the secondary feature substantially slowed responses (494 vs. $544 \mathrm{~ms}$ ). However, the interaction was nonsignificant, $F$ $(1,14)=0.5, p=.49$, indicating that changes of the visual secondary feature alone (without any change in the motor sequence) did not cause PRCs.

Confounded-repetitions analysis The standard analysis, in which changes of the response covaried with changes of the response-defining feature, revealed both main effects and the interaction to be significant. Changes of the primary feature led to slower RTs than did repetitions (522 vs. $510 \mathrm{~ms}), F(1,14)=$ $5.3, p<.05, \eta_{\mathrm{p}}{ }^{2}=.27$, and changes of the secondary feature/ response also slowed responses (494 vs. $538 \mathrm{~ms}$ for repetitions vs. changes), $F(1,14)=32.5, p<.001, \eta_{\mathrm{p}}{ }^{2}=.69$. The interaction was also significant: $F(1,14)=5.7, p<.05, \eta_{\mathrm{p}}{ }^{2}=.30$.

\section{Discussion}

Changes of the primary visual feature (color/form) in the paradigm with four response-relevant features interacted with combined changes of the response and the responsedefining feature, replicating the standard interaction (in contrast to Yashar \& Lamy, 2011, who found this interaction to be only marginally significant in two experiments).

Crucially, this interaction vanished when we analyzing only sequences in which the response repeated and the response-defining feature could either repeat or change. At the same time, the interaction was significant when analyzing only sequences in which the response-relevant feature changed and the response could either repeat or change. This indicated that the relevant factor that drives PRCs is the sequence of motor responses, rather than the sequence of the response-relevant feature. While this is in line with Yashar and Lamy's (2011) conclusion, the present findings go beyond theirs, in that we used a nonsearch, single-item discrimination task instead of a standard compound task. That is, the primary feature, changes of which caused PRCs, were target-defining - and thus task-relevant-in Yashar and Lamy's study; by contrast, in the present Experiment Experiment 2 (and in Exp. 1b), the primary feature (the target's color and form) was completely task-irrelevant. Consequently, PRCs could be observed as an interaction between the sequences of completely task-irrelevant features and of the motor response. In terms of the MWS hypothesis, this means that the PRCs observed in compound-search tasks are not necessarily related to the search component of these tasks, but rather to the subsequent discrimination component.

\section{Arithmetical simulation}

The MWS hypothesis (see the introduction) assumes that observed RT sequence effects result from multiple modulations of performance at different stages of processing (Rangelov et al., in press). Specifically, main effects for the change versus repetition of the primary (target-defining) and, respectively, the secondary (response-defining) feature, as well as their interaction, originate from different processing stages, while ultimately yielding one, superimposed RT data pattern. Thus, the MWS hypothesis can also explain how the RT interactions between primary- and secondaryfeature changes/repetitions can differ in form among different studies. Especially, as is suggested by the label "partialrepetition costs," one might expect the interaction to be symmetric: full changes and full repetitions of primary and secondary features should yield equally fast responses, and partial changes should yield equally slow RTs. Frequently, however, the fastest RTs occur with full repetitions, while the other three conditions yield equally slow responses (e.g., Töllner et al., 2008, or the present Exp. 1a). According to the MWS hypothesis, however, observable RT intertrial effects are superpositions of several intertrial effects originating at different processing stages. To illustrate this, we simulated RT interactions between changes versus repetitions of primary and secondary features. RTs could be 
affected (1) by changes/repetitions of the primary feature, (2) by changes/repetitions of the secondary feature, and (3) by their interaction. Let the four possible intertrial transition conditions be denoted $\mathrm{sP}-\mathrm{sS}, \mathrm{dP}-\mathrm{sS}, \mathrm{sP}-\mathrm{dS}$, and $\mathrm{dP}-\mathrm{dS}$, where "s" and "d" denote "same" and "different," and "P" and "S" denote the "primary" and "secondary" features (e. g., dP-dS means "different primary feature and different secondary feature on the current trial $n$, relative to the preceding trial $n-1$ "). Then, for these four conditions, RTs are calculated by adding the RT costs for changing the primary visual feature, changing the secondary visual feature, and changing only one of the two features. Thus, the transition condition $\mathrm{dP}-\mathrm{SS}$ involves a change of the primary feature (producing a primary cost), a repetition of the secondary feature (generating no secondary cost), ${ }^{1}$ and a partial change (producing a PRC):

$\mathrm{sPsS}=0+0+0$

$\mathrm{dPsS}=$ primary cost $+\mathrm{PRC}$

$\mathrm{sPdS}=\mathrm{PRC}+$ secondary cost

$\mathrm{dPdS}=$ primary cost + secondary cost

That is, the main effects (MEs) of repeating the primary or secondary feature, $\mathrm{ME}_{\text {pri }}$ and $\mathrm{ME}_{\mathrm{sec}}$, can be calculated as

$M E_{p r i}=\frac{d P s S+d P d S}{2}-\frac{s P s S+s P d S}{2}=$ primary cost

$M E_{s e c}=\frac{s P d S+d P d S}{2}-\frac{s P s S+d P s S}{2}=$ secondary cost

For illustrating the four basic types of interactions (Eqs. 1-4), the PRCs and primary and secondary costs were set to be of the same size. As can be seen in Fig. 5, the interaction between changes of the primary and secondary features depends on what additional intertrial effects are added to the superposition: Effects related to the secondary feature keep the slopes of the interaction intact but shift the two lines relative to each other, whereas effects related to the primary feature affect the slopes of the two lines.

Note that the computational scheme above is intended as one step to formalize how the MWS account can be applied

\footnotetext{
${ }^{1}$ The terms "primary cost" and "secondary cost" in this article are only used as shorthands for "change costs arising from changes of the primary/secondary feature" and do not indicate any other ordering (temporal or otherwise)
}

to existing problems in the literature (in this case, how the interaction between the sequence of the primary visual feature and that of the secondary visual features or the [selected/executed] response can take on different forms in an interaction plot). For instance, the present scheme does not make statements about the processing stages at which primary costs, secondary costs, and PRCs arise. According to the MWS hypothesis, all types of cost can originate from one or more processing stages. Primary costs, for instance, can arise at one or more of the following stages: target selection (as proposed by the dimension-weighting account), target identification (Huang et al., 2004), and/or discrimination of the secondary feature. Formally, this could be stated as:

primary cost $=\sum_{i \in S}$ primary $\cos t_{i}$,

where $S$ is the set of all possible processing stages from which sequences of the primary feature result in a cost to processing performance.

The present experiments, however, set several constraints on the composition of these costs. For instance, the fact that, in Experiment 1a, primary costs were smaller on cued than on noncued trials, but were still present on cued trials, and the fact that primary costs were observed in Experiments $1 \mathrm{~b}$ and Experiment 2, in which no search was necessary at all, indicate that primary costs arise not only at processing stages related to the search component, but also at those related to the discrimination component of compound tasks. According to the MWS account, the same logic also applies to PRCs: They can stem from the search and/or the discrimination component of compound tasks. The present data falsify accounts according to which PRCs do not originate from the discrimination component (e.g., Huang et al., 2004). However, they cannot definitely rule out the possibility that a portion of PRCs also stem from the search component of compound tasks. Nevertheless, searchdriven PRCs appear rather unlikely: In contrast to primary costs, which interacted with cue in Experiment 1a, PRCs did not interact significantly with cue. Additional constraints on the processing stage(s) or task component(s) from which a particular cost arises are posed by the electrophysiological findings of Töllner et al. (2008), as well as those from the task switch paradigm of Rangelov et al. (2011a, 2011b, in press).

\section{General discussion}

From the present finding of PRCs - in terms of an interaction between changes (vs. repetitions) of the target color/ form and the response-critical feature (stripe orientation)even when the search component of a compound task was effectively removed, four specific conclusions could be 
Fig. 5 Simulated partialrepetition costs (PRCs) for four conditions. First, at top left are pure PRCs, without any further superposed intertrial effects. At bottom left, PRCs and a response repetition effect. At top right, PRCs and an intertrial effect of the primary feature. Finally, at bottom right are PRCs and both a response repetition effect and an intertrial effect of the primary feature
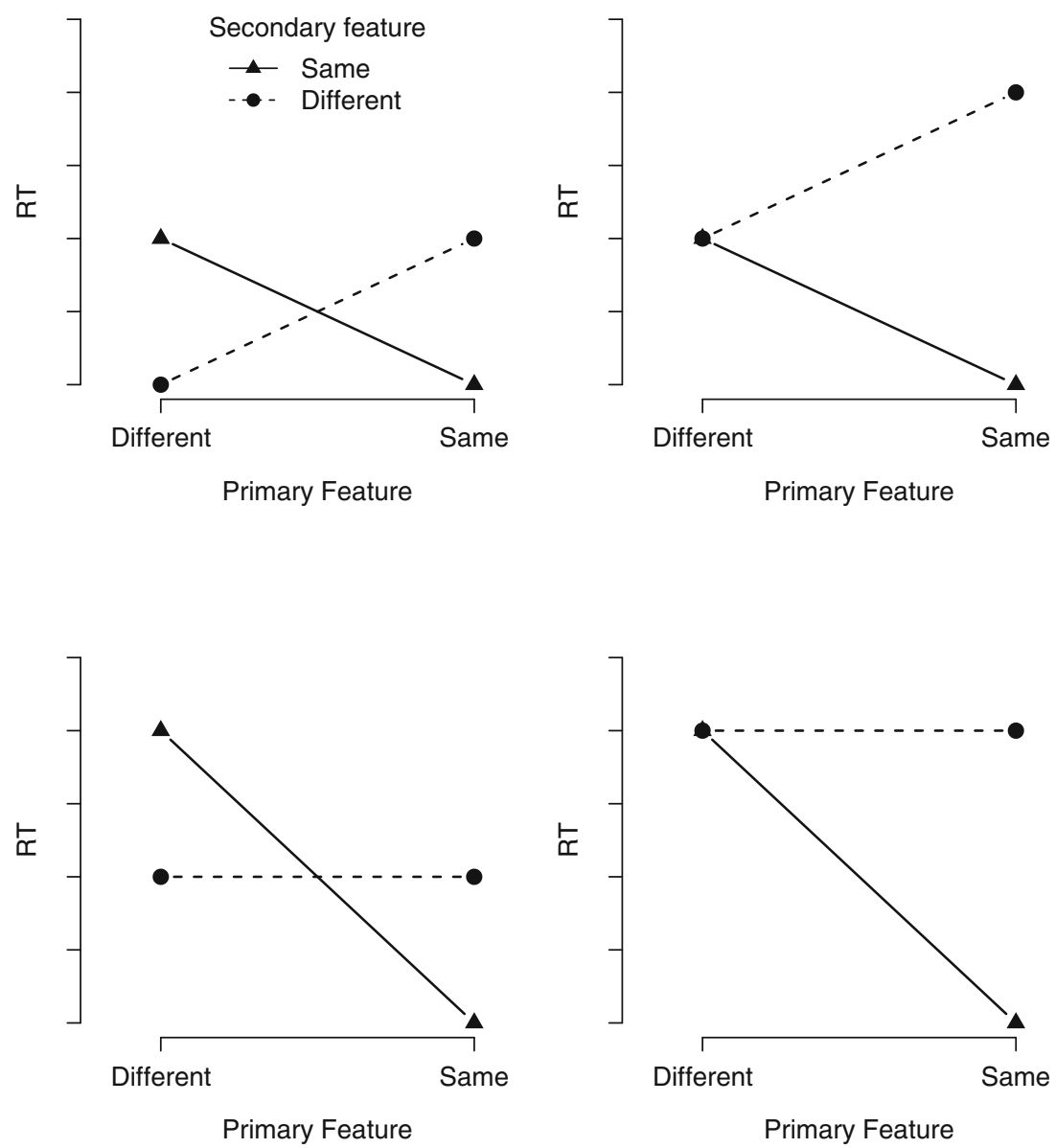

drawn: (1) PRCs in compound tasks do indeed stem from the discrimination, rather than exclusively from the search, component of compound-search tasks; this is consistent with the account of Töllner et al. (2008), but at variance with that of Huang et al. (2004). (2) PRCs are independent of search (in particular, target verification) processes. (3) PRCs do not require that the primary feature (here, color/ form) be in any way task-relevant-for example, for guiding the search for the target. (4) The sequence of motor responses, rather than of the response-relevant features, is what is responsible for PRCs. In addition, we have provided a schematic mathematical formulation of the MWS hypothesis, which explains how different forms of the RT interaction between changes of the primary feature and response actually arise.

\section{Unitary versus multiple sources of intertrial effects}

The present findings support the view that RT-priming effects in visual search do not stem from a single mechanism, but instead arise from multiple "memory traces" influencing multiple processing stages (Kristjánsson \& Campana, 2010; Lamy et al., 2010; Rangelov et al., 2011a, b, in press). Consequently, there is no such thing as the intertrial-priming RT effect, but rather, observed RT effects are actually the superposition of several priming effects.

The logic of multiple memory systems (producing intertrial effects at different processing stages) also suggests a caveat as to the conclusions that can be drawn from the present data: Although they falsify models that assume that PRCs are generated exclusively by processes involved in searching for a target, and indicate instead that PCRs originate from the subsequent discrimination stage, they do not rule out that search itself may also lead to PRCs. Conceivably, there may also exist a mechanism in the search component of compound tasks that is sensitive to changes in target- and response-defining features. However, Lamy et al. (2010) observed that, when the target and nontarget features in a compound-search task change dynamically, either early or late within a single trial episode, "priming of pop-out" (Maljkovic \& Nakayama, 1994) is evident only for early changes, whereas PRCs are observed only for late changes. This dissociation favors the conclusion that PRCs are solely generated at the discrimination (rather than the search) component of compound tasks.

Overall, support for the MWS hypothesis stems from several sources of evidence: electrophysiological, manipulation of task demands, carryover of intertrial effects across different tasks, and temporal manipulation of target-defining features. 
First, as reviewed above, there is an electrophysiological dissociation of intertrial effects in ERPs, reflecting attentional selection and response execution (Töllner et al., 2008). Second, removal of the search component in compound tasks leads to the disappearance of some components of intertrial effects (Goolsby \& Suzuki, 2001), whereas others also remain present in the absence of search (Mortier et al., 2005, and the present study). Third, it has been demonstrated that intertrial effects can be observed across changes of tasks, but only if the two tasks in sequence share processing requirements, either in terms of search (e.g., detection and localization) or of discrimination demands (single-item feature discrimination and target/ nontarget discrimination). For task sequences that do not share task requirements (e.g., detection and single-item feature discrimination), no intertrial effects survive task switches (Rangelov et al., 2011b, and the present study).

Additional evidence for the MWS hypothesis is provided by the present Experiment 1a, in which changes of the primary feature (i.e., dimension changes) produced larger effects when the trial did not, relative to when it did, contain a cue. That is, when observers had to search for the target, dimension change effects were increased, as compared to when the target location was indicated by a $100 \%$-valid precue. This effect is largely in line with the dimensionweighting account, according to which the search process is facilitated by dimension repetitions-leading to repetition benefits on search trials, but not on nonsearch trials. However, the fact that a significant benefit remained even on the latter trials indicates that main effects related to the repetition/change of a primary visual feature are not confined to the search process per se. Consistent with this, we found a main effect of repeating versus changing the primary feature, even for the single-item tasks used in Experiments $1 \mathrm{~b}$ and Experiment 2 (as well as for the nonsearch trials of Exp. 1a), despite the fact that the primary feature was completely taskirrelevant under these conditions (see also Kahneman, Treisman, \& Gibbs, 1992).

\section{Conclusion}

In summary, the present findings support the hypothesis that PRCs do not stem from searching for a target, but from the discrimination component of compound tasks. Specifically, they reflect the interaction between changes of a taskirrelevant feature and the preparation (or execution) of a response. Additionally, they provide further behavioral evidence that the assumption of a unitary mechanism responsible for sequence effects in visual-search paradigms is untenable; rather, multiple sequence-sensitive mechanisms operating at different stages of processing (preattentive, postselective, and motor-related) contribute to repetition effects, measured in terms of RTs. Our study has also demonstrated that a microanalysis of task requirements and of the resulting cognitive-processing stages, as suggested by the MWS account, is a fruitful heuristic tool to resolve debates about the role of implicit memory in visual search.

Author note This research was supported by a grant from Deutsche Forschungsgemeinschaft (DFG) Cluster of Excellence: Cognition for Technical Systems (Grant EC 142 to H.J.M. and M.Z.), DFG Grant ZE 887/3-1 (to M.Z. and H.J.M.), and Grant FOR480 from the DFG Research Group (to H.J.M.).

\section{References}

Ásgeirsson, Á. G., \& Kristjánsson, Á. (2011). Episodic retrieval and feature facilitation in intertrial priming of visual search. Attention, Perception, \& Psychophysics, 73, 1350-1360. doi:10.3758/ s13414-011-0119-5

Becker, S. I. (2010). Oculomotor capture by colour singletons depends on intertrial priming. Vision Research, 50, 2116-2126. doi:10.1016/j.visres.2010.08.001

Bravo, M. J., \& Nakayama, K. (1992). The role of attention in different visual-search tasks. Perception \& Psychophysics, 51, 465-472. doi:10.3758/BF03211642

Bundesen, C. (1990). A theory of visual attention. Psychological Review, 97, 523-547. doi:10.1037/0033-295X.97.4.523

Chan, L. K. H., \& Hayward, W. G. (2009). Feature integration theory revisited: Dissociating feature detection and attentional guidance in visual search. Journal of Experimental Psychology: Human Perception and Performance, 35, 119-132. doi:10.1037/00961523.35.1.119

Duncan, J. (1985). Visual search and visual attention. In M. I. Posner \& O. S. M. Marin (Eds.), Attention and performance XI (pp. 85106). Hillsdale, NJ: Erlbaum.

Eimer, M. (1996). The N2pc component as an indicator of attentional selectivity. Electroencephalography and Clinical Neurophysiology, 99, 225-234. doi:10.1016/0013-4694(96)95711-9

Found, A., \& Müller, H. J. (1996). Searching for unknown feature targets on more than one dimension: Investigating a "dimensionweighting" account. Perception \& Psychophysics, 58, 88-101. doi:10.3758/BF03205479

Goolsby, B. A., \& Suzuki, S. (2001). Understanding priming of colorsingleton search: Roles of attention at encoding and "retrieval. Perception \& Psychophysics, 63, 929-944. doi:10.3758/ BF03194513

Hackley, S. A., \& Valle-Inclán, F. (2003). Which stages of processing are speeded by a warning signal? Biological Psychology, 64, 2745. doi:10.1016/S0301-0511(03)00101-7

Hillstrom, A. P. (2000). Repetition effects in visual search. Perception \& Psychophysics, 62, 800-817. doi:10.3758/BF03206924

Huang, L., Holcombe, A. O., \& Pashler, H. (2004). Repetition priming in visual search: Episodic retrieval, not feature priming. Memory \& Cognition, 32, 12-20. doi:10.3758/BF03195816

Kahneman, D., Treisman, A., \& Gibbs, B. J. (1992). The reviewing of object files: Object-specific integration of information. Cognitive Psychology, 24, 175-219. doi:10.1016/0010-0285(92)90007-O

Kingstone, A. (1992). Combining expectancies. Quarterly Journal of Experimental Psychology, 44A, 69-104. doi:10.1080/ 14640749208401284

Kristjánsson, Á., \& Campana, G. (2010). Where perception meets memory: A review of repetition priming in visual search tasks. Attention, Perception, \& Psychophysics, 72, 5-18. doi:10.3758/ APP.72.1.5 
Lamy, D., Yashar, A., \& Ruderman, L. (2010). A dual-stage account of inter-trial priming effects. Vision Research, 50, 1396-1401. doi:10.1016/j.visres.2010.01.008

Maljkovic, V., \& Nakayama, K. (1994). Priming of pop-out: I. Role of features. Memory \& Cognition, 22, 657-672. doi:10.3758/ BF03209251

Mortier, K., Theeuwes, J., \& Starreveld, P. (2005). Response selection modulates visual search within and across dimensions. Journal of Experimental Psychology: Human Perception and Performance, 31, 542-557. doi:10.1037/0096-1523.31.3.542

Müller, H. J., \& Krummenacher, J. (2006). Locus of dimension weighting: Preattentive or postselective? Visual Cognition, 14, 490-513. doi: $10.1080 / 13506280500194154$

Olivers, C., \& Meeter, M. (2006). On the dissociation between compound and present/absent tasks in visual search: Intertrial priming is ambiguity driven. Visual Cognition, 13, 1-28. doi:10.1080/ 13506280500308101

Rangelov, D., Müller, H. J., \& Zehetleitner, M. (2011a). Dimensionspecific intertrial priming effects are task-specific: Evidence for multiple weighting systems. Journal of Experimental Psychology: Human Perception and Performance, 37, 100-114. doi:10.1037/ a0020364
Rangelov, D., Müller, H. J., \& Zehetleitner, M. (2011b). Independent dimension-weighting mechanisms for visual selection and stimulus identification. Journal of Experimental Psychology: Human Perception and Performance, 37, 1369-1382. doi:10.1037/a0024265

Rangelov, D., Müller, H. J., \& Zehetleitner, M. (in press). The multiple-weighting-systems hypothesis: Theory and empirical support. Attention, Perception, \& Psychophysics. doi:10.3758/ s13414-011-0251-2

Töllner, T., Gramann, K., Müller, H. J., Kiss, M., \& Eimer, M. (2008). Electrophysiological markers of visual dimension changes and response changes. Journal of Experimental Psychology: Human Perception and Performance, 34, 531-542. doi:10.1037/0096-1523.34.3.531

Wolfe, J. M. (1994). Guided Search 2.0: A revised model of visual search. Psychonomic Bulletin \& Review, 1, 202-238. doi:10.3758/ BF03200774

Yashar, A., \& Lamy, D. (2011). Refining the dual-stage account of intertrial feature priming: Does motor response or response feature matter? Attention, Perception, \& Psychophysics, 73, 2160 2167. doi:10.3758/s13414-011-0182-y

Zehetleitner, M., \& Müller, H. J. (2010). Salience from the decision perspective: You know where it is before you know it is there. Journal of Vision, 10(14), 35. doi:10.1167/10.14.35 